\title{
Stress and strain rate analysis of the FT4 Powder Rheometer
}

\author{
Colin Hare $^{1 *}$, and Mojtaba Ghadiri $^{2}$ \\ ${ }^{1}$ Department of Chemical and Process Engineering, The University of Surrey, Guildford, UK \\ ${ }^{2}$ School of Chemical and Process Engineering, The University of Leeds, Leeds, UK
}

\begin{abstract}
The Freeman FT4 Powder Rheometer has been reported to describe well the powder flow behaviour in instances where other techniques fail. We use DEM to simulate the FT4 operation for slightly cohesive large glass beads at a range of strain rates. The curved impeller is shown to be beneficial in comparison to a flat blade as the variation of shear stress across the blade is reduced. The shear stress in front of the blade correlates well with flow energy (which the device measures) for a range of tip speeds and is shown to increase approximately linearly with tip speed when operating beyond the quasi-static regime.
\end{abstract}

\section{Introduction}

Poor understanding and control of powder flowability is a major cause of process downtime and reduction in process performance. The onset of flow in a quasi-static state is largely understood, with methods available for design of storage bins and hoppers [1]. However many processes operate at higher strain rates and are concerned with dynamic flow, such as mixing and die filling. The only commercially available devices which characterise powder flow in the dynamic regime are the FT4 Powder Rheometer of Freeman Technology and that of AntonPaar. Here we address the analysis of the dynamics of the FT4, whilst that of Anton-Paar has been reported by Salehi-Kahrizsangi et al. [2]. In the FT4 a twisted-blade impeller is rotated and driven downwards into a column of powder. The device directly measures the torque on the blade, $T$, and the force on the base of the vessel, $F_{\text {base }}$, in order to determine 'flow energy',

$$
E_{\text {flow }}=\int_{0}^{h}\left(\frac{T}{R \tan \alpha}+F_{\text {base }}\right) d h
$$

where $h$ is the penetration depth, $R$ the radius of the impeller and $\alpha$ the helix angle to the horizontal of the impeller movement. The FT4 measurement has often been cited to correlate with powder flow performance in processes, however the meaning of flow energy and how it can be used to better design or control powder processes is not well understood. Here we use DEM to simulate the FT4 device operation in order to determine the stress and strain distribution in the bed under a range of operational speeds.

\section{Stress analysis}

The FT4 operation is simulated using EDEM (DEM Solutions), using spheres of $1.7-2.1 \mathrm{~mm}$ diameter. The linear elastic-plastic and adhesive contact model of Pasha et al. [3] is used to represent cohesive spheres with a surface energy of $29 \mathrm{~mJ} / \mathrm{m}^{2}$, in order to correlate with the experimental work of Hare et al. [4]. The particle-particle sliding and rolling friction coefficients are set at 0.3 and 0.01 , respectively, to best represent the experimental glass beads in their work. The spheres are generated in a $50 \mathrm{~mm}$ diameter vessel and allowed to settle under gravity, after which the particles whose centre is greater than $80 \mathrm{~mm}$ above the base are removed, thus leaving approximately 25,000 particles. The bed diameter and height are identical to the experimental setup.

The $48 \mathrm{~mm}$ diameter impeller is rotated in an anticlockwise direction and driven downwards into the bed using a tip speed of $100 \mathrm{~mm} / \mathrm{s}$ and helix angle of $5^{\circ}$ to the horizontal, until a penetration depth of $70 \mathrm{~mm}$ is reached, $10 \mathrm{~mm}$ above the base of the bed.

The normal and shear stresses in measurement cells in front of the blade (Figure 1) are estimated using Eq 2

$$
\sigma_{i j}=\frac{1}{V} \sum_{1}^{N} F_{i j} \cdot r
$$

where $V$ is the cell volume, $N$ is the number of particles in the cell, $F$ is the force acting in direction $i$ on face $j$ [4], using Cartesian coordinates. The principal stresses are then determined from the eigenvalues of these nine stress tensors, and the deviatoric (shear) stress,

* Corresponding author: c.hare@surrey.ac.uk 


$$
\tau_{D}=\frac{\sqrt{\left(\sigma_{1}-\sigma_{2}\right)^{2}+\left(\sigma_{1}-\sigma_{3}\right)^{2}+\left(\sigma_{2}-\sigma_{3}\right)^{2}}}{\sqrt{6}}
$$

where $\sigma_{1}, \sigma_{2}$ and $\sigma_{3}$ are the major, intermediate and minor principal stresses, respectively. The hydrostatic (confining) stress, $\sigma_{H}$, is taken as the average of the three principal stresses. (a)

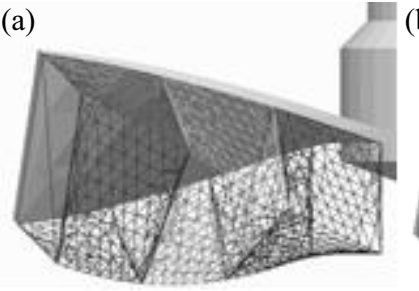

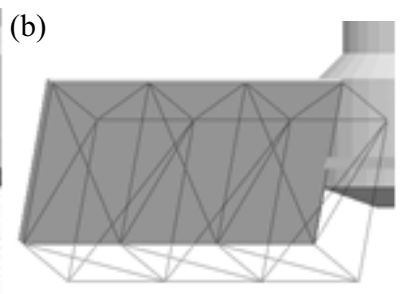

(b)
Fig. 1. Measurement cells (a) curved blade, (b) flat blade

Hare et al. [4] showed that the shear stress in front of the curved blade is approximately constant across its radial length. Here we analyse the dynamics of a flat blade set at the same inclination angle with respect to the horizontal plane as the tip of the real, curved blade (Fig. 1), by DEM simulation. Figure 2 shows the 10-point moving average of the deviatoric stress against penetration depth for these two blades. It can be seen that with the flat blade the stress increases with radial position across the blade, from approximately 90 to 160 $\mathrm{Pa}$ at the final penetration depth of $70 \mathrm{~mm}$. In contrast, the stresses near the shaft and tip of the curved blade at this penetration depth show much less deviation, with values of 100 and $120 \mathrm{~Pa}$, respectively.

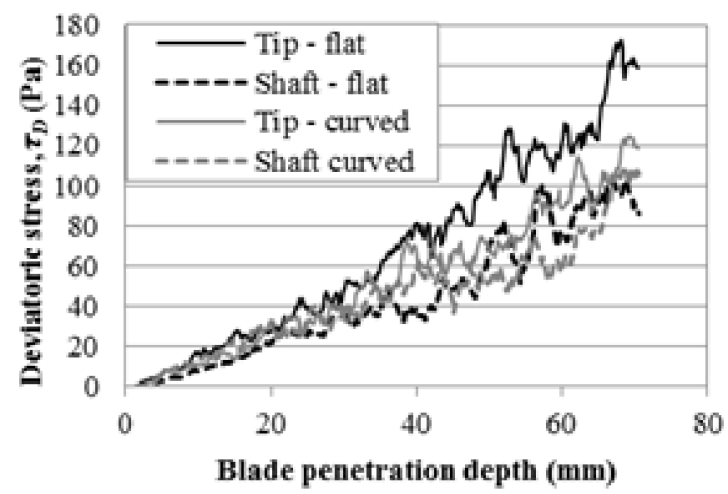

Fig. 2. Shear stress against penetration depth for curved and flat blades

For some powders the flow energy is observed to change with tip speed. The FT4 is capable of operating up to a tip speed of $300 \mathrm{~mm} / \mathrm{s}$. Using the curved blade we simulate the FT4 operation using tip speeds of 40 $1200 \mathrm{~mm} / \mathrm{s}$, with a fixed helix angle of $5^{\circ}$. In all cases the shear stress is found to be approximately constant across the blade length, and increasing linearly with penetration depth (though notable scatter is observed as in Fig. 2). A linear regression is applied to the shear stress against penetration depth, to estimate the maximum shear stress occurring in front of the blade, $\tau_{\max }$. Figure 3 shows the maximum shear stress and flow energy for the range of impeller speeds tested.

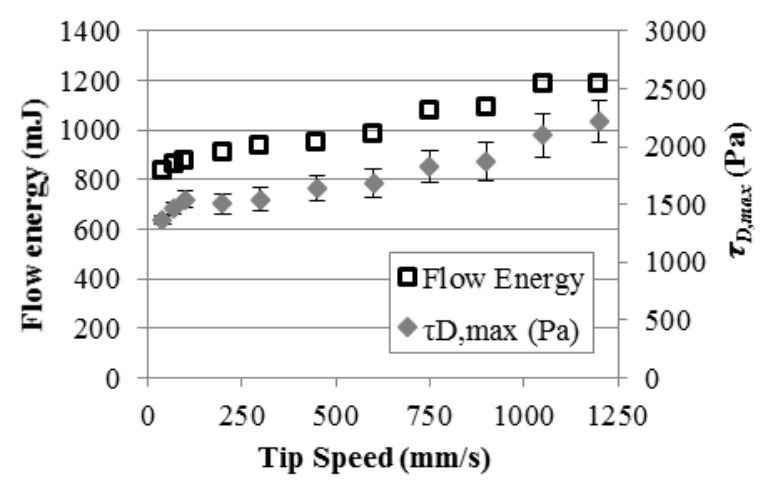

Fig. 3. Maximum shear stress and flow energy for various impeller tip speeds

The maximum shear stress and the flow energy increase with tip speed through the entire range tested. The shear stress error bars represent one standard deviation from the linear regression of shear stress against blade penetration depth (e.g. Figure 2), at maximum penetration depth. The error bars indicate that the increase in shear stress with impeller speed is statistically significant. There is a strong correlation between flow energy and shear stress for the range of impeller speeds tested.

\section{Strain rate analysis}

In addition to the stress profile in the bed, there is a need to understand the strain rate regime of operation in the FT4 tests. Figure 4 shows the particle velocity distribution for impeller tip speeds, $v_{t i p}$, of 70,600 and $1050 \mathrm{~mm} / \mathrm{s}$, at a penetration depth of $10 \mathrm{~mm}$. The darkest colour represents the tip speed and the lightest is $10 \%$ of the tip speed. At an impeller speed of $70 \mathrm{~mm} / \mathrm{s}$ the bed is densely packed and a small fraction of the bed is mobilised. As the impeller speed is increased there is a clear dilation of the bed, with particles being lifted high above the initial bed height at impeller speeds of 600 and $1050 \mathrm{~mm} / \mathrm{s}$.

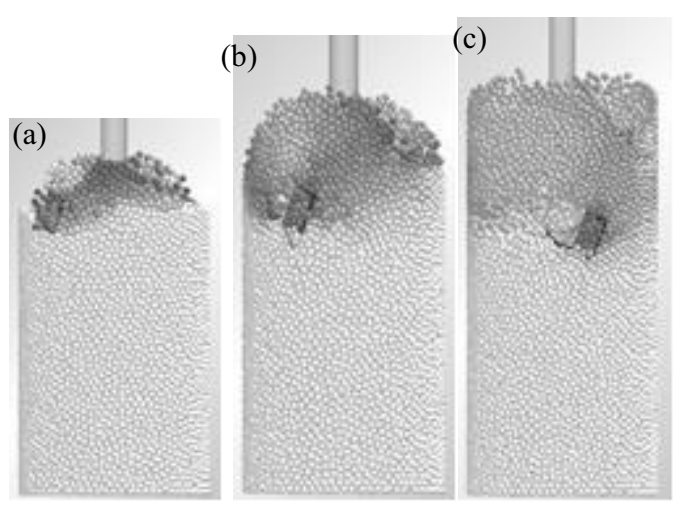

Fig. 4. Particle velocities within the bed at a penetration depth of $10 \mathrm{~mm}$ with impeller tip speeds (a) $70 \mathrm{~mm} / \mathrm{s}$ (b) $600 \mathrm{~mm} / \mathrm{s}$ and (c) $1050 \mathrm{~mm} / \mathrm{s}$ 


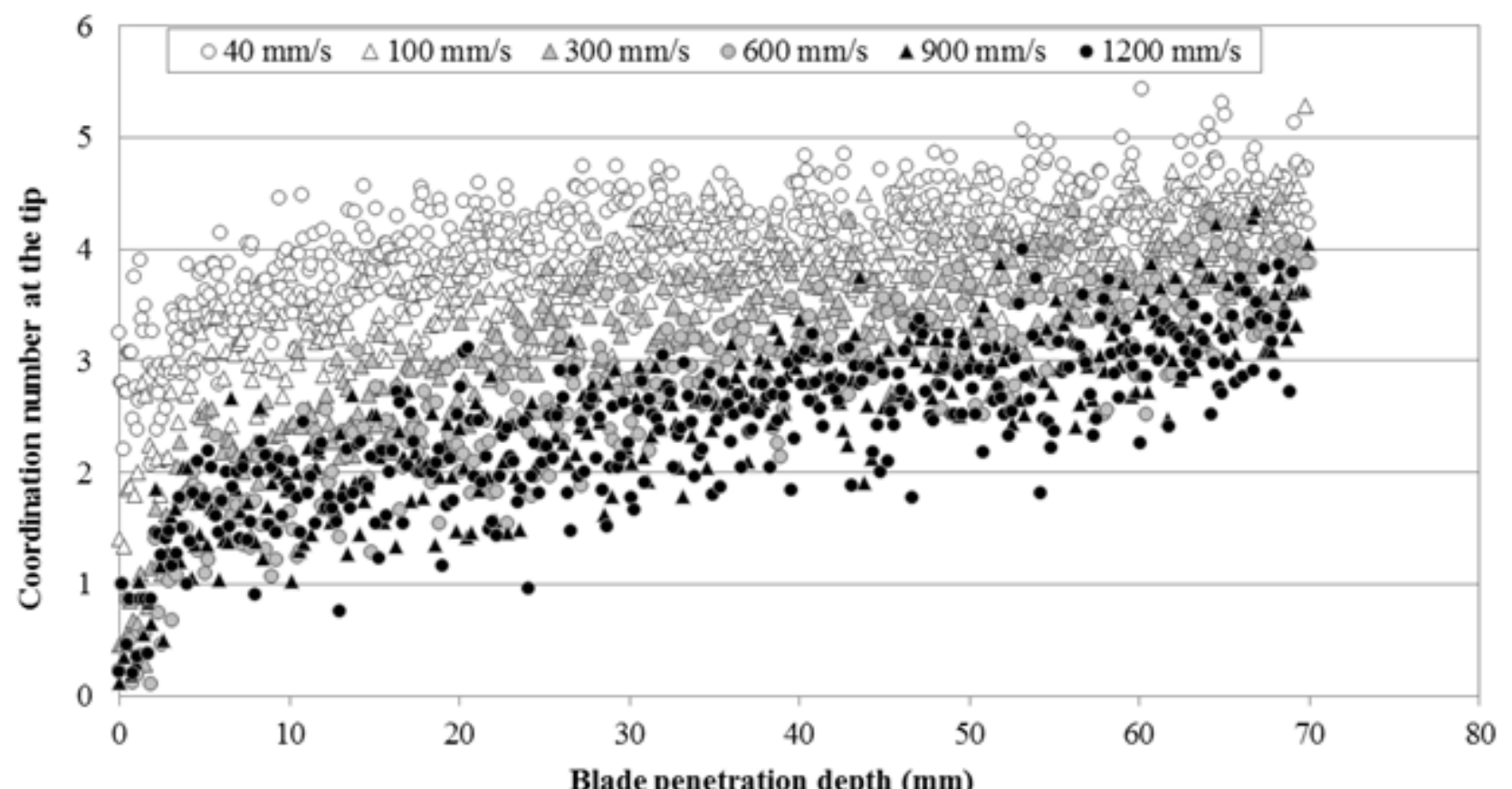

Fig. 5. Coordination number in the measurement cell near the tip for impeller speeds of 40, 100, 300, 600, 900 and $1200 \mathrm{~mm} / \mathrm{s}$

The evolution of coordination number, $Z$, within the measurement cell near the tip during the FT4 test with a range of impeller speeds is shown in Figure 5. For all impeller speeds the coordination number increases with penetration depth. At penetration depths of a few $\mathrm{mm}$ the increase in $Z$ with depth is sharp, particularly at greater impeller speeds. This may be largely due to an increasing number of particles being lifted and dispersed high above the bed. At penetration depths of $10 \mathrm{~mm}$ (as shown in Figure 4) and beyond, there is a more gradual increase in $Z$ with penetration depth. This is expected to be partly due to the previously lifted powder settling on top of the bed; resisting the upward movement of particles out of the measurement cell, in addition to the fact that as the active region of the bed descends, it is under an increasing hydrostatic stress due to the weight of the bed above. At an impeller speed of $40 \mathrm{~mm} / \mathrm{s} Z$ is approximately 4-4.5 at a penetration depth of $70 \mathrm{~mm}$. An increase of impeller speed to $100 \mathrm{~mm} / \mathrm{s}$ causes negligible change in $Z$, whereas at an impeller speed of $300 \mathrm{~mm} / \mathrm{s}$ there is notable change as $Z$ drops below a value of 4. As the impeller speed is increased to 1200 $\mathrm{mm} / \mathrm{s}$ there is a continual drop in $Z$.

The length of a shear band is estimated from Figure 4 by considering the number of particles running parallel to the blade from the last particle with velocity $v_{t i p}$ to the first particle with velocity $0.1 v_{\text {tip }}$, above and below the blade. The strain rate, $\gamma$, is calculated using Eq 4 .

$$
\gamma=d v /\left(n_{\text {band }} \cdot d_{p}\right)
$$

where $d v$ is the velocity differential across the shear band $\left(0.9 v_{\text {tip }}\right), n_{\text {band }}$ is the number of particles in the shear band and $d_{p}$ is the average particle diameter. The strain rate is calculated using this approach at penetration depths of 25, 38, 51 and $64 \mathrm{~mm}$, and the average taken. For all impeller speeds the particles immediately in front of the blade have maximum velocity, equal to the tip speed. The dimensionless strain rate, $\gamma^{0}$, introduced by Tardos et al. [6] is calculated using Eq 5.

$$
\gamma^{0}=\gamma \sqrt{d_{p} / g}=\frac{d v}{n_{\text {band }}} \sqrt{\frac{1}{d_{p} \cdot g}}
$$

where $g$ is gravitational acceleration.

The strain rates at the given tip speeds are shown in Table 1. It is suggested that at low dimensionless strain rates the bed is in the quasi-static regime and beyond values of $0.15-0.25$ the system is in the intermediate regime [6], thus implying that at the maximum tip speed of the FT4 $(300 \mathrm{~mm} / \mathrm{s})$, the bed is in this regime. It is worth noting from Eqs $4 \& 5$ that if the number of particles in a shear band remains constant, a reduction in particle size leads to an increase in dimensionless strain rate, thus suggesting that for fine particles the FT4 may be capable of operating in the dynamic regime. However, for cohesive powders the number of particles in the shear band may increase due to clustering, so further work is needed to investigate this.

The flow regime is commonly assessed by the inertial number,

$$
I=\gamma \cdot d_{p} \sqrt{\rho / \sigma_{H}}
$$

where $\rho$ is the particle density of the bed. Figure 6 shows the inertial number in the measurement cell near the tip of the blade against penetration depth for a range of impeller speeds. In all cases the inertial number decreases as the impeller penetrates further into the bed, with an initially rapid decline followed by a more gradual reduction beyond a depth of 5-10 mm. The bed is in the dense flow regime up to an impeller speed of $900 \mathrm{~mm} / \mathrm{s}\left(1 \times 10^{-3}<I \leq 1 \times 10^{-1}\right)$, whilst at greater 
impeller speeds the bed is in the collisional flow regime $\left(I>1 \times 10^{-1}\right)$. The regime boundaries differ significantly between the inertial number approach and that of Tardos et al. [6], with the inertial number approach suggesting the system enters the dense and collisional flow regimes at much lower impeller speeds.

Table 1. Strain rates at various tip speeds

\begin{tabular}{|c|c|c|c|}
\hline $\begin{array}{c}\text { Impeller } \\
\text { speed } \\
(\mathbf{m m} / \mathbf{s})\end{array}$ & $\begin{array}{c}\text { Average } \\
\boldsymbol{n}_{\text {band }}\end{array}$ & $\begin{array}{c}\text { Strain rate, } \\
\boldsymbol{\gamma}\left(\mathbf{s}^{\mathbf{1}}\right)\end{array}$ & $\begin{array}{c}\text { Dimensionless } \\
\text { strain rate, } \boldsymbol{\gamma}^{\mathbf{0}}\end{array}$ \\
\hline 40 & 4.8 & 4.6 & 0.06 \\
\hline 70 & 4.1 & 8.6 & 0.12 \\
\hline 100 & 4.4 & 12 & 0.17 \\
\hline 200 & 4.8 & 23 & 0.32 \\
\hline 300 & 4.3 & 37 & 0.51 \\
\hline 450 & 4.8 & 57 & 0.79 \\
\hline 600 & 4.9 & 75 & 1.04 \\
\hline 750 & 4.6 & 99 & 1.38 \\
\hline 900 & 4.8 & 100 & 1.42 \\
\hline 1050 & 5.4 & 110 & 1.58 \\
\hline 1200 & 5.4 & 130 & 1.75 \\
\hline
\end{tabular}

\section{Conclusions}

The FT4 Powder Rheometer operation was simulated using DEM with slightly cohesive spheres of approximately $2 \mathrm{~mm}$ diameter. The curved blade of the FT4 is shown to minimise the variation of shear stress across the blade length, as compared to a flat blade of the same inclination angle. The shear stress at maximum penetration depth is shown to correlate well with the flow energy for a wide range of impeller tip speeds. The shear stress and the flow energy both increase slightly with tip speed throughout the entire range of impeller speeds tested. Beyond a tip speed of $300 \mathrm{~mm} / \mathrm{s}$ the bed is found to be in the intermediate/dense flow regime using the approach of Tardos et al. [6]. It is noteworthy that if the number of particles in a shear band is independent of particle size, the impeller tip speed corresponding to this flow regime boundary is expected to reduce as particle size is reduced. In contrast, the inertial number approach suggests that even at a tip speed of $40 \mathrm{~mm} / \mathrm{s}$ the bed is in the dense flow regime, and enters the collisional regime at a tip speed beyond $900 \mathrm{~mm} / \mathrm{s}$.

\section{Acknowledgements}

The authors would like to express their thanks to Messrs Tim Freeman and Jamie Clayton of Freeman Technology, UK, for their support of this work, and Dr Marty Murtagh of Corning inc. for useful discussions.

\section{References}

1. A.W. Jenike, Powder Technol., 1, 237-244 (1967).

2. H. Salehi-Kahrizsangi, D. Shütz, D. Barletta, M. Poletto, AIChE Annual Meeting, Nov 13-18, San Francisco, USA (2016).

3. M. Pasha, S. Dogbe, C. Hare, A. Hassanpour, M. Ghadiri, Granul. Matter, 16, 151-162 (2014).

4. C. Hare, U. Zafar, M. Ghadiri, T. Freeman, J. Clayton, M.J. Murtagh, Powder Technol., 285, 123127 (2015).

5. K. Bagi, Mech. Mater., 22, 165-177 (1996).

6. G.I. Tardos, S. McNamara, I. Talu, Powder Technol., 131, 92-98 (2003).

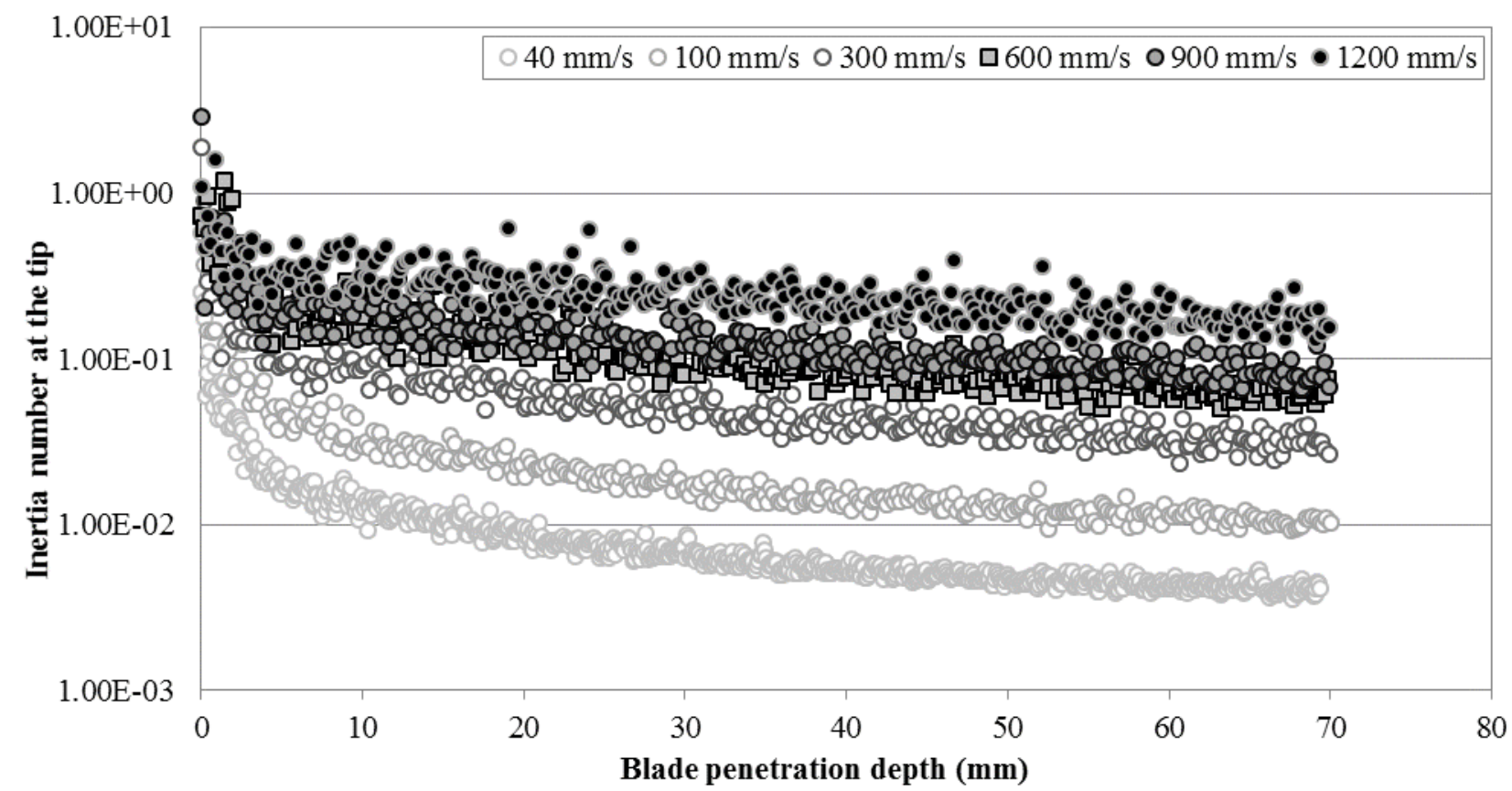

Fig. 6. Inertia number in the measurement cell near the tip for impeller speeds of 40, 100, 300, 600, 900 and $1200 \mathrm{~mm} / \mathrm{s}$ 